\title{
An E-commerce enterprise's business CRM problems and countermeasures
}

\author{
Cuican Wang \\ School of Beijing Jiaotong University, Beijing 100044, China \\ 15125397@bjtu.edu.cn
}

\begin{abstract}
In recent years, with the continuous development of social economy, the continuous improvement of information technology, e-commerce has penetrated into every aspect of people's lives, we enter a new age of electronic commerce. In such a rapid development of online shopping scenario, An e-commerce enterprise if only by attracting new business customers to increase revenue, increase market share, and overcome a number of competitors is not enough, we must also pay attention to prevention of the old customers drain. So carry out the business customer-centric customer relationship management for an e-commerce enterprise becomes imminent.
\end{abstract}

Keywords: customer relations; management; business enterprise; customer segmentation.

\section{Introduction}

With the development of market economy, the technological level slowly close, product homogeneity, the trend is clear. Thus, by the simple products to win market model obsolete. So far, with the support of advanced information technology under, CRM has penetrated into various industries and has been rapidly gaining popularity. An e-commerce enterprise as a late start of the e-commerce business, which is in the business model of $\mathrm{C} 2 \mathrm{C}$, want to win a share in a number of competitors, and acquire long-term development, it needs to be converted into its business model to customer-centric.

\section{Statement of problem}

Because e-commerce model is very convenient, which greatly meet the modern society, people's fast-paced lifestyle, so more and more e-commerce businesses have sprung up, while another form of war had been produced between business malls.

In an increasingly competitive situation, e-commerce enterprise want to continue to develop, expand, not just the pursuit of high-quality products or services, should also include customer. Understand and grasp customer demand trends, continue to attract more customers while strengthening and maintaining existing customer relationships is particularly important.

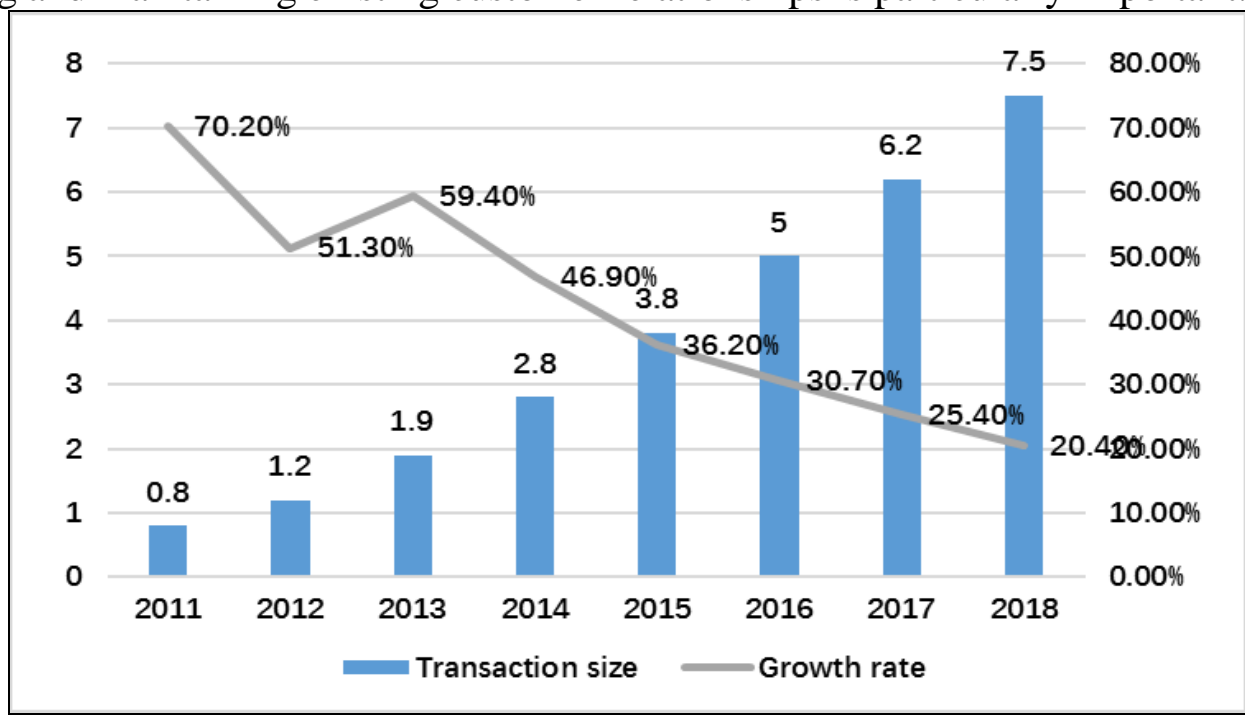

Fig. 1 China online shopping market transaction size in 2011-2018 
Shown above is the Chinese industry information network issued theOnline shopping market research and trends report, which demonstrated 2011-2018 years' ${ }^{6}$ China's online shopping market transactions situation.

As can be seen from the figure 1, the size of China's online shopping transactions since 2011 has been showing growth situation, and to continue the growth trend continue. In the past 2015, the Chinese e-commerce market size is 16.4 trillion yuan, has been an increase of $22.7 \%$, including online shopping market transactions reached 3.8 trillion yuan, an increase of $36.2 \%$. According to the National Bureau of Statistics released data show that in 2015 total sales reached 30.1 trillion yuan, online shopping in total retail sales of social consumer goods accounted for $12.6 \%$, an increase of $2 \%$ over 2014, promoting economic development, an important force in the market.

\section{An E-commerce enterprise Customer Relationship Management Problems and Causes}

For An e-commerce enterprise, to find the main problems existing in the current customer relationship management, and to find the causes of the problems of the existing problems, it is an important prerequisite for an e-commerce enterprise to carry out customer relationship management in the future.

\subsection{Customer acquisition difficult Problems and cause analysis.}

Internet technology is increasingly updated today, An e-commerce enterprise still rely on the traditional client acquisition mode, so customer information was not comprehensive enough or cannot meet the business needs, in addition, the company has no scientific method for analyzing customer data, so the enterprise cannot measure customer true value, it cannot produce its guiding role of customer relationship management.

After the introduction of the customer, the promotion sector had not been timely following-up of the customer's feedback, so that a number of potential customers lost in vain.

The traditional customer acquisition is come out from the customer's point of view, the enterprise through a variety of ways to improve potential customers' interest and understanding of the products and services which they offered, making them active-site consulting, purchase [1]. An e-commerce enterprise current customer acquisition is one such way. And another perspective, customer acquisition occurs between businesses and their customers, not only from the perspective of the customer to look at this issue, but also from a business perspective, that companies should have access to customer information, thereby reducing the range between potential customers and target customers, saving business costs.

\subsection{Customer segmentation Problem and cause analysis.}

An e-commerce enterprise in the initial stage to focus on branding and technology development, ignore the customer relationship management and marketing. Now, there are many customer resources, but still very limited.

Customer classification criteria of each company's business center is not uniform, for lack of a comprehensive understanding of the customer situation. And the enterprise doesn't have a scientific Customer identification method. The reason why so weak in the customer segment link, is by its overall operating model decisions [2].

\subsection{Customer relationship maintenance Problem and cause analysis.}

Although An e-commerce enterprise has been still stressed "customer-centric" in recent years, but the idea was not well implemented in practice,

The Marketing model of An e-commerce enterprise is that each client has a corresponding marketing during the customer relationship continues to be accountable, especially for large customers, on the surface it seems to be a kind of "one to one" marketing strategy, but in the actual operation merely to serve the needs of an individual customer, but not truly realize customized service to each customer or the same type of customer demand for personalized, so that they cannot understand the real needs of most customers, and therefore cannot get the trust of customers, We lost the opportunity to establish a relationship of interdependence with customers, thus affecting the 
growth of business turnover. It appears to reduce employee satisfaction and loyalty and so on. E-commerce industry is highly competitive, customer resources among peers snatch going on all the time, since some of the personal conduct of former employees, lead to the disclosure of information and the loss of customers, resulting in customer relationship management had a negative impact.

\subsection{Churn Problem and cause analysis.}

An e-commerce enterprise presents flat organizational structure now, power is too concentrated on the Business Center, while at the same time, they lack of regulatory mechanisms to the center principal, appears not high employee satisfaction, loyalty and decreased phenomenon. Electricity supplier industry is highly competitive, customer resources among peers snatch going on all the time, because of personal behavior of certain former employees, lead to the disclosure of information on corporate customers, loss of customers, so the company's customer relationship management had a negative impact [3].

\section{Suggestions and reflection of an e-commerce enterprise's customer relationship management}

The problem of an e-commerce enterprise's customer relationship management, mainly in organizational structure, customer acquisition, customer segmentation and customer relationship maintenance aspects. These issues are cross-cutting and complex, and thus cannot be solved overnight. Combined with the actual situation of An e-commerce enterprise and the operability of the measures, the article propose countermeasure from two aspects of customer segmentation and human resources management to improve the level of customer information management.

\subsection{Customer segmentation based on customer value.}

The main problem of an e-commerce enterprise is that the customer classification criteria of each business center is not uniform, nor a customer identification model suited to their own needs. More intense market competition so that an e-commerce enterprise customer life cycle length of the uncertainty is further intensified. Therefore, this article divides the entire customer life cycle length into the known customer life cycle length as well as the anticipated customer life cycle length [4]. Accordingly, the customer life cycle value is divided into customer's historical value, customer current value and customer potential value, the three most.

And then on this basis, according the customer value to do the customer segmentation. There is no uniform model for customer segmentation, and companies often according to their needs to conduct customer segmentation. In this paper, according to the particularity of the A e-commerce enterprise, using the improved algorithm of K-means clustering algorithm--K-mad algorithm to the enterprise business customer segmentation, in order to achieve effective customer segmentation $[5,6]$.

The main flow of the K-mad algorithm has the following three steps:

Step one: using the density distribution of the regional space to determine the value of the data object clustering

Step two: the new clustering of the centroid of the class as the initial cluster center

Step three: the algorithm is used to get the clustering results.

The key of the algorithm is step one-how to determine the distribution of high-density region by the number density region of space, the clusters $\mathrm{K}$ value is determined by the geometrical features of the data objects distribution rather than simply on the basis of experience. Clustering results are as follows Table 1.

Table 1 Customer Segments Results Table 


\begin{tabular}{|c|c|c|c|c|}
\hline $\begin{array}{c}\text { Cluster ing } \\
\text { category }\end{array}$ & $\begin{array}{c}\text { Number of } \\
\text { customers }\end{array}$ & $\begin{array}{c}\text { Monthly average } \\
\text { transaction amount }\end{array}$ & $\begin{array}{c}\text { Monthly average number } \\
\text { of transactions }\end{array}$ & Proportion \\
\hline C1 & 31 & 139729 & 2813 & 0.010881 \\
\hline C2 & 152 & 48261 & 595 & 0.053352 \\
\hline C3 & 542 & 19392 & 1579 & 0.190242 \\
\hline C4 & 2031 & 3710 & 961 & 0.712882 \\
\hline C5 & 93 & 301 & 248 & 0.032643 \\
\hline
\end{tabular}

From customer segmentation results point of view, class $\mathrm{C} 1$ customers are An e-commerce enterprise's best quality customer class. Class C2 customers are potential customers of quality. C3 class customers belong to the general category of large customers. Class C4 customers are the majority of small and medium retail customers. Class c5 customers belong to sleep customer class. And the classification results are basically in accordance with Pareto's law.

\subsection{Human resource management.}

An e-commerce enterprise employee flow rate and the loss rate is larger, indicates that the employee satisfaction is not high, this will greatly affect the customer relationship management work smoothly; the quality of sales staff is directly related to the level of customer relationship maintenance work, so employees need to change the concept of customer relationship management. Enable employees to combine personal interests with the company's goals and make a good career plan for employees. It should also adopt fair and effective means of encouragement.

An e-commerce enterprise marketing staff remuneration not only directly linked with the investment capacity, should also be associated with subsequent customer relationship management, customer feedback, complaints and return work should be used as the reference indexes of employee earnings, make employee self-improving customer relationship management ability, so as to improve the An e-commerce enterprise customer satisfaction, help to maintain customer relationship.

\section{Summary}

This article through analyze the issues in customer relationship management that an e-commerce enterprise is facing now, so as to put forward the improvement measures. Mainly put forward the following two measures, one is based on the analysis of the life cycle of customer value, and then use the K-mad algorithm to improve the customer segmentation; the second one is a more rational allocation of resources and personnel, in order to improve the customer churn management. Through the above method, hoping to effectively improve an e-commerce enterprise customer relationship management.

\section{References}

[1] Wang Xiqiu. Research on Customer Value and Its Evaluation [J]. Nankai Business Review. 2005, $8(5)$

[2] Guo Chonghui, Zhao Zuowei. Customer Segmentation and Change Mining Based on Customer Behavior for 4S Shop [J]. Journal of Industrial Engineering and Engineering Management. 2015, 29(4)

[3] Luo Xinxing, Deng Li, Zhao Yujie. Decision support system for customer value prediction of new product based on CBR and MAHP [J]. Computer Integrated Manufacturing Systems. 2014, $20(10)$

[4] Chen Mingliang. A Study of the Customer Life Cycle Model [J]. Jorunal of Zhejiang University (Humanities and Social Science). 2002, 32 (6): 66-72. 
[5] Xiang Jianchi, Liu Xiangbin, Zi Wucheng. Research on K-Means clustering algorithm based on density and its application to customer segmentation [J]. Computer Engineering and Applications. 2008, 44(35): 246-248.

[6] Sun Ke, Lie Jie, Wang Xueying. K Mean Cluster Algorithm with Refined Initial Center Point [J]. Journal of Shenyang Normal Universitu (Natural Science Edition).2009, 27 (4):448-450. 